\title{
CYP450 y farmacogenética en Guatemala. Revisión narrativa
}

\author{
CYP450 and pharmacogenetics in Guatemala. Narrative review
}

\author{
Rodrigo J. Vargas (D) 1,2,3, Oscar M. Cobar (DiD) 1,2,4
}

${ }^{1}$ Escuela de Estudios de Postgrado, Facultad de Ciencias Médicas, Universidad de San Carlos de Guatemala ${ }^{2}$ Red Latinoamericana de Implementación y Validación de pautas de farmacogenómica clínica (Relivaf-Cyted) ${ }^{3}$ Universidad Galileo, ${ }^{4}$ Universidad del Istmo

*Autor al que se dirige la correspondencia: rodrigovargas@galileo.edu

\section{Resumen}

Recibido: 28 de junio 2020 / Revisión: 11 de febrero 2021 / Aceptado: 09 de junio 2021

E 1 proyecto HapMap ha generado información y preguntas sobre la diversidad genética en las distintas poblaciones del mundo. En las últimas décadas, proyectos como la elucidación del genoma del mestizo mexicano han revelado las distancias genéticas entre mestizos y amerindios en México. Cerca de 20 genes son actualmente estudiados en paneles comerciales asociados al metabolismo de fármacos, uno de ellos el gen que expresa la enzima $C Y P 2 C 19$, la cual metaboliza cerca de 26 fármacos de importancia clínica. El objetivo fue revisar la literatura científica en Google Scholar, PubMed y ScienceDirect que reporta resultados sobre estudios farmacogenéticos en Guatemala, otros que presentan hallazgos sobre distancias genéticas en el guatemalteco y se compara con lo que se conoce de otras poblaciones del continente y el mundo, haciendo énfasis en CYP2C19. El mestizaje en Guatemala fue único, por ello es importante investigar sus variantes alélicas asociadas al metabolismo de fármacos, para permitir una terapéutica más efectiva y segura que mejore la calidad de vida del guatemalteco.

Palabras claves: Amerindios, farmacogenética, enzimas metabolizadoras de fármacos, CYP450

\begin{abstract}
$\mathrm{T}$ he HapMap project has generated information and queries about genetic diversity in the different populations around the world. In recent decades, research projects such as the elucidation of the genome of the Mexican Mestizo, have exposed the genetic distances between mestizos and Amerindians in Mexico. About 20 genes are currently studied in commercial panels associated with drug metabolism. One of them CYP2C19, that expresses the CYP2C19 enzyme, that metabolizes about 26 drugs of clinical importance. The objective was to review the scientific literature in Google Scholar, PubMed and ScienceDirect that reports results on pharmacogenetic studies in Guatemala, others that presented findings over genetic distances in Guatemala, as well as a comparison with the knowledge about other populations of the continent and world, with emphasis in CYP2C19 gen. Miscegenation in Guatemala was unique and is important to investigate the Guatemalan allelic variants associated with drug metabolism to allow a more effective and safe therapeutic and improve their quality of life.
\end{abstract}

Keywords: Amerindians, Pharmacogenetics, drug metabolizing enzymes, CYP450 


\section{Introducción}

Las técnicas de Biología Molecular como herramientas de la Farmacogenética, son el presente en la Farmacología. La búsqueda de la adhesión a un tratamiento farmacológico de pacientes a quienes se les administra combinaciones de fármacos es compleja, por lo que es importante conocer información genética que coadyuve a mejorar la calidad y seguridad de la terapéutica, hasta alcanzar dosis personalizadas de medicamentos (Hewett et al., 2002).

Una de las familias de enzimas más importantes en el metabolismo de los fármacos es el complejo citocromo p450 (CYP450), el que consta de cerca de 80 miembros que, en conjunto, metabolizan más del $90 \%$ de los fármacos aprobados por la Food and Drug Administration de los Estados Unidos y actualmente comercializándose. La región del gen CYP2C19 expresa a la familia de enzimas $C Y P 2 C 19$, que metaboliza cerca del $10 \%$ de los fármacos en el mercado, algunas benzodiacepinas y antidepresivos como el clopidogrel, citalopram y escitalopram, entre otros (Zhang et al., 2020).

Sin embargo, polimorfismos en estas regiones genéticas, implican la expresión de variantes alélicas de la enzima, lo que repercute en el metabolismo de los fármacos, haciéndolos ineficientes o tóxicos a dosis convencionales y poco eficaces (Gonzalez et al., 2003)

En 2012 se publica la creación de la base de datos The Pharmacogenomics Knowledgebase (PharmGKB), un recurso para estudiar el impacto de la variación genética humana en la respuesta del organismo a la administración de medicamentos. PharmGKB proporciona información clínicamente relevante, como pautas de dosificación, anotaciones en etiquetas de los medicamentos y las asociaciones de genes/fármacos potencialmente procesables, así como las relaciones genotipo-fenotipo. Adicionalmente, permite determinar asociaciones de variantes genéticas y medicamentos, utilizando criterios bien definidos, basados en revisión cuidadosa de la literatura (Whirl-Carrillo et al., 2012).

Actualmente, la mayoría de estudios gen/fármaco se han llevado a cabo en poblaciones norteamericanas, europeas y asiáticas, sus resultados han permitido comprobar la variabilidad en la genética de sus poblaciones, comparándolas con otras. Resultados de estos estudios, muestran que la población guatemalteca comparte similitudes con el resto de la población latinoamericana (Garrido et al., 2013).

En 2011 en Memphis, Estados Unidos, se implementó el servicio de farmacogenética clínica en el
St. Jude Children's Research Hospital, en la que farmacéuticos resolvieron consultas, incluyendo interpretación de resultados de estudios farmacogenéticos y brindaron recomendaciones de aplicación terapéutica, obteniéndose muy buena aceptación por parte del sector médico (Crews, 2011).

A nivel centroamericano, en 2015, se publicó un estudio farmacogenético realizado en voluntarios sanos centroamericanos, que mostró diferencias en la frecuencia de algunos biomarcadores farmacogenéticos y por ende, variabilidad étnica entre la población centroamericana y otras poblaciones latinoamericanas (Céspedes-Garro et al., 2015).

Para CYP2C19 existen diversos estudios farmacogenéticos, siendo el más cercano a Guatemala, el que se realizó en occidentales mexicanos-mestizos, obteniéndose una frecuencia de $6 \%$ y $4 \%$ respectivamente para metabolizadores pobres (MP) y ultrarrápidos (MUR) (Salazar-Flores et al., 2012). Posteriormente, en un estudio de casos y controles también en México, la frecuencia estimada de MP de CYP2C19 fue del $8 \%$ en la población general (Martínez-Quintana et al., 2017). De igual forma en un estudio reciente da frecuencias estimadas de CYP2C19*1 y *2 fue de $91.6 \%$ y $8.4 \%$ respectivamente en voluntarios sanos de la ciudad de México, mientras que $C Y P 2 C 19 * 3$ no se encontró (León-Moreno et al., 2019). En Panamá, un estudio de CYP2C19 en sus grupos amerindios, no encontró individuos MP en la población cuna de Panamá (Isaza et al., 2006).

Esta revisión se centra en los metabolizadores pobres del CYP2C19 y sus estudios recientemente realizados en el continente y poblaciones ancestrales, así como estudios en Guatemala. Las bases Google Scholar, PubMed y ScienceDirect fueron consultadas con las palabras clave, CYP2C19, farmacogenética y Guatemala. Cuarenta artículos fueron encontrados y 11 fueron seleccionados, además fueron consultados artículos con temas de distancia genética, amerindios y países como México, Centroamérica y redes financiadas por el Programa Iberoamericano de Ciencia y Tecnología para el Desarrollo (CYTED), como la Red Latinoamericana de Implementación y Validación de Pautas de Farmacogenómica Clínica (RELIVAF) y la Red Iberoamericana de Farmacogenética y Farmacogenómica (RIBEF), redes colaborativas para generación de conocimiento en farmacogenética en Latinoamérica. Los artículos publicados antes de 2002 fueron excluidos, artículos en inglés y español fueron incluidos. 


\section{Contenido}

\section{Genes asociados a metabolismo de fármacos}

Cerca de 35 genes son determinados en la actualidad en paneles comerciales, la mayoría con interés preventivo en tratamientos farmacológicos (Haga \& Kantor, 2018). Todos estos genes son de importancia según el tratamiento farmacológico del paciente, sin embargo, son los que expresan las enzimas de la familia del CYP450 los más importantes, debido entre otros, a que realiza el metabolismo de xenobióticos en el organismo (Al Omari \& Murry, 2007).

La naturaleza polimórfica de estos genes ha sido relacionada con la respuesta a la administración de fármacos, es el caso del CYP450, ya que ello determina como el organismo los metaboliza y el efecto (positivo o negativo) de los fármacos (Ingelman-Sundberg et al., 2007).

Se conocen numerosas enzimas que catalizan el metabolismo de fármacos de fase I. El hallazgo en la década de 1980, de que la hidrólisis del relajante muscular succinilcolina por la enzima butirilcolinesterasa estaba genéticamente condicionado (Levano et al., 2005), constituyó uno de los hitos que marcaron el desarrollo de la Farmacogenética. A partir de este descubrimiento, se han reportado numerosos ejemplos de variaciones farmacogenéticas clínicamente relevantes en las que intervienen enzimas metabolizadoras de reacciones de tipo I. Al respecto, una de las primeras enzimas estudiadas y de actividad mejor conocida, es la tiopurina S-metiltransferasa (TPMT) actuando sobre la azatioprina (Relling et al., 2019).

\section{Citocromo P450}

La respuesta del organismo a la administración de fármacos, es consecuencia de la interacción entre el fármaco y sus enzimas metabolizadoras, siendo CYP450 la más ampliamente estudiada en sus genes que la expresan, lo que permite una comparación entre los diferentes grupos poblacionales y las frecuencias de variantes alélicas que poseen.

Una cantidad cada vez mayor de entidades farmacológicamente activas, tienen la capacidad de interaccionar inhibiendo o estimulando el sistema enzimático CYP450 (Manikandan \& Nagini, 2018).

Los fármacos son xenobióticos, metabolizados por las enzimas expresadas por las regiones de genes 1A1, 1A2, 2A6, 2A13, 2B6, 2C8, 2C9, 2C18, 2C19,
2D6, 2E1, 2F1, 3A4, 3A5, 3A7, sin embargo, solo las familias enzimáticas CYP450 1, 2 y 3 se asocian al metabolismo de medicamentos, estando descritas en humanos 57 familias para CYP450. Si bien los fármacos pueden ser metabolizados por otras enzimas fuera del sistema CYP450, el 70\% son metabolizados por CYP450. Esta familia de enzimas es altamente polimórfica y su distribución depende del grupo poblacional (Zanger \& Schwab, 2013). A pesar de la conocida fuerte asociación entre CYP450 y la respuesta farmacológica, continúa el estudio de alelos aún no conocidos y su selectividad hacia fármacos, lo que mejorará la predicción de respuestas farmacológicas basadas en genotipos (Shah et al., 2016).

\section{CYP2C19}

Una familia de enzimas CYP450 es la CYPC19, de la cual se han identificado más de 35 variantes alélicas (www.pharmvar.org), sin embargo, no todas son frecuentes en las poblaciones y las frecuencias pueden variar en una población a otra. Estudios indican que el alelo $C Y P 2 C 19 * 1$ el primero que se identificó, es el más frecuente, seguido de alelos $* 2, * 3$ y en algunos casos un alelo asociado a respuesta ultrarrápida CYP2C19*17 (Wedlund, 2000).

El alelo CYP2C19*2 es originado por un defecto de splicing en el exón 5, generando un cambio en la posición 681 de una guanina en lugar de una adenina (Ibeanu et al., 1998) y es el alelo más frecuentemente asociado a un metabolizador pobre. Sus efectos ocasionan cambios en guías de práctica clínica en la administración de fármacos, donde esfuerzos como el clinical pharmacogenetics implementation consortium -CPIC(cpicpgx.org), han permitido el desarrollo de guías de práctica clínica y protocolos para alrededor de 26 fármacos que son afectados en su metabolismo por el alelo *2. Sin embargo, estos esfuerzos son principalmente derivados de estudios en caucásicos, lo que ha motivado la creación de redes en Latinoamérica con el mismo propósito, tal es el caso de la red RELIVAF del CYTED.

La Farmacogenética se inicia en 1979 con el estudio de la deficiencia de la biotransformación de la metionina (Kupfer \& Preisig, 1984), luego ha avanzado a la elucidación de la enzima responsable por estudios en tres dimensiones y entendimiento y predicción de posibles sustancias orgánicas con los diferentes alelos del CYP2C19 (Deguchi et al., 2019).

Existe una pregunta sin responder de interés clínico sobre los cambios en CYP2C19 con la edad. 
Si bien existen planteamientos sobre los cambios que pueden existir (Xie et al., 2002), aún no existe una respuesta concluyente.

\section{Metodologías para el estudio de la farmacogenética}

Las técnicas para identificación de variantes alélicas del CYP540 así como para la determinación de paneles de varios marcadores como mutaciones de fibrosis quística, tiopurina s-metiltransferasa, marcadores relacionados con anticoagulantes, analgesia, entre otros, son cada vez más utilizados en guías de práctica clínica, incluyendo información farmacogenética (Duong et al., 2020). Las técnicas varían entre desde PCR-RFLP descrita ya en estudios farmacogenéticos realizados en Guatemala (Xajil Ramos et al., 2020), hibridación inversa, prometedora técnica de fácil implementación (Gialeraki et al., 2010), hasta técnicas más modernas y complejas como Afimetrix (Gonzalez-Covarrubias et al., 2016), luminex (Spierings \& Dunbar, 2013), MALDI-TOF (Schaeffeler et al., 2008), PsychArray (Martínez-Magaña et al., 2020) y también secuenciación de nueva generación (NGS por sus siglas en Inglés), que permite identificar las mutaciones y nuevas variantes alélicas aún no conocidas (Gaedigk et al., 2018). Esta última es la técnica que permitiría a Guatemala obtener el mapa de la diversidad genómica del guatemalteco a partir de la elucidación de genomas completos, como México en 2009 (Silva-Zolezzi et al., 2009). También es importante incluir análisis bioinformático para determinar el impacto funcional de las variaciones genéticas. Herramientas como VEP, PROVEAN y FATHMM han resultado útiles en farmacogenética (Gonzalez-Covarrubias et al., 2019).

\section{Estudios en poblaciones}

Resultados de investigaciones sobre las frecuencias alélicas de CYP2C19 en cada grupo poblacional y su aplicación, ha sido una pregunta recurrente, evidenciada en la cantidad cada vez mayor de artículos sobre frecuencias alélicas de metabolizadores pobres asociados a CYP2C19. Un estudio paradigmático fue el realizado en las islas Vanuatú en 1999 (Kaneko et al., 1999). El estudio fue uno de los más extensos realizados hasta el momento $(n=5,538)$ y reveló que el $78 \%$ de la población poseía alelos de metabolizadores pobres para el $C Y P 2 C 19$. Esto planteó la pregunta sobre la necesidad de conocer más sobre las frecuencias alélicas en las poblaciones no caucásicas. Hasta el momento, las más ampliamente estudiada en el mundo.

Las diferencias para CYP2C19 en el alelo *2 (MP) más frecuente, son en general distintas; hispanos $12.6 \%$ asiáticos $34.5 \%$ y europeos $15.1 \%$ (Mirzaev et al., 2017). Los resultados han llevado a investigaciones en América en población mestiza y amerindia, donde resalta el estudio realizado en Bolivia, un país con porcentajes altos de población amerindia, donde sus frecuencias alélicas resultaron ser más altas para el alelo CYP2C19*2 que la de caucásicos (Bravo-Villalta et al., 2005).

Otro avance significativo en el entendimiento del efecto de las frecuencias alélicas en mestizos y amerindios lo aportó México en 2012 (Salazar-Flores et al., 2012), donde se evaluaron cinco grupos de amerindios, resaltando el grupo tarahumara con un 31\% para el alelo $C Y P 2 C 19^{*} 2$, un valor sin duda alto en comparación con mestizos mexicanos.

El alelo $C Y P 2 C 19 * 3$ un metabolizador pobre, es poco frecuente en Latinoamérica. En Costa Rica el primer estudio poblacional sobre CYP2C19 fue realizado en 2016 y si bien, incluyen poblaciones ancestrales, resalta la presencia del alelo*17, un MUR frecuente en la población afrocaribeña y la ausencia de *3 en todas sus poblaciones estudiadas (Céspedes-Garro et al., 2016). En el occidente mexicano fue reportado un $8.61 \%$ y $14.29 \%$ para alelos $* 2$ y $* 17$ en mestizos, sin presencia del alelo *3 (Favela-Mendoza et al., 2015).

El mestizaje es distinto en cada país latinoamericano como producto de su historia y éste puede ser distinto aún dentro de un mismo país. En Venezuela la diferencia para $C Y P 2 C 19$ en un heterogéneo grupo mestizo y el grupo ancestral warao fue demostrado en 2017 (Flores-Gutiérrez et al., 2017). Los grupos amerindios son objeto de análisis y comparación respecto a su respuesta farmacogenética, sin embargo, la información es aún insuficiente y los datos se interpretan como una población que no difiere de otros grupos mestizos. Estas diferencias dependen en gran medida del aislamiento del grupo ancestral (Leitão et al., 2020). Sin embargo, esto resulta cierto para CYP2D6, pero estudios que incorporan $C Y P 2 C 9$ y $C Y P 2 C 19$ concluyen en una mayor frecuencia de alelo salvaje $* 1$ en grupos amerindios y diferencias importantes entre los grupos ancestrales en Latinoamérica (Naranjo et al., 2018). Resalta también el estudio realizado por MartínezMagaña y colaboradores en México, en el que identifican los patrones de mestizaje con el objetivo de mejorar la consejería farmacogenética en dicho país (2020). 


\section{Farmacogenética en Guatemala}

La pregunta sigue siendo: ¿Cuál es la frecuencia de MP en Guatemala?, un país con alta población de origen ancestral.

Hasta 2015, un consorcio regional, la RIBEF, realizó estudios sobre frecuencias alélicas en el continente americano, destacando una revisión de Céspedes-Garro y colaboradores en 2016, en dónde estudios realizados en Guatemala sobre CYP450 no habían sido publicados, no así la importancia clínica farmacogenética y farmacovigilancia en Latinoamérica (Sosa-Macías et al., 2016).

Resulta importante conocer si existe presencia de alelo $C Y P 2 C 19 * 3$ en Guatemala, el cual en México solo fue encontrado en menos del $1 \%$ de mestizos y no fue encontrado en amerindios (Salazar-Flores et al., 2012).

La composición de la población mestiza de Guatemala se considera en equilibrio y su composición ancestral es compleja, sin embargo, su distancia frente a grupos amerindios de Guatemala es tan cercana que es posible afirmar que el mestizaje en Guatemala tiene una muy alta composición amerindia (MartinezEspin et al., 2006).

La Red-Consorcio apoyada por CYTED, RELIVAF, generó un estudio sobre Cáncer de Colon y farmacogenética en Latinoamérica (López-Cortéz et al., 2019). Sin embargo, Guatemala no fue incluida en el estudio. A partir de 2019 Guatemala fue incluida en el Consorcio, lo que supone un aumento de información en farmacogenética para Guatemala en el futuro cercano.

\section{Conclusión}

La Farmacogenética en Guatemala está poco estudiada y desarrollada. No existen evidencias de estudios poblacionales que arrojen información sobre las frecuencias alélicas en los distintos grupos étnicos para CYP450 en el país. Sin embargo, estudios en Latinoamérica con poblaciones ancestrales, subrayan y enfatizan la necesidad de realizar investigaciones de índole similar en Guatemala, fundmentalmente por sus distancias genéticas respecto a otros grupos poblacionales. Las poblaciones mayoritarias de Guatemala, principalmente aquellas aisladas, junto con el mestizo son prioritarias a investigar farmacogeneticamente.

\section{Agradecimientos}

Se agradece a Armando Cáceres por su valiosa colaboración y el apoyo brindado.

\section{Contribución de los autores}

Coordinación, elaboración y revisión del Documento: todos los autores.

Participación en análisis de datos, estructura y en la escritura del documento: todos los autores.

\section{Referencias}

Al Omari, A., \& Murry, D. J. (2007). Pharmacogenetics of the cytochrome P450 enzyme system: review of current knowledge and clinical significance. Journal of Pharmacy Practice, 20(3), 206-218. https://doi.org/10.1177/ 0897190007304821

Bravo-Villalta, H. V., Yamamoto, K., Nakamura, K., Bayá, A., Okada, Y., \& Horiuchi, R. (2005). Genetic polymorphism of CYP2C9 and CYP2C19 in a Bolivian population: An investigative and comparative study. European Journal of Clinical Pharmacology, 61(3), 179-184. https://doi.org/ 10.1007/s00228-004-089-5

Céspedes-Garro, C., Naranjo, M., Ramírez, R., Serrano, V., Fariñas, H., Barrantes, R., \& Llerena, A. L. (2015). Pharmacogenetics in Central American healthy volunteers: Interethnic variability. Drug Metabolism and Personalized Therapy, 30(1), 19-31. https://doi.org/10.1515/dmdi-2014-0025

Céspedes-Garro, C., Rodrigues-Soares, F., JiménezArce, G., Naranjo, M. E. G., Tarazona-Santos, E., Fariñas, H., Barrantes, R., \& Llerena, A. (2016). Relevance of the ancestry for the variability of the Drug-Metabolizing Enzymes CYP2C9, CYP2C19 and CYP2D6 polymorphisms in a multiethnic Costa Rican population. Revista de Biología Tropical, 64(3), 1067-1076. https://doi.org/ 10.15517/rbt.v64i3.20901

Crews, K. R. (2011). Development and implementation of a pharmacist-managed. American Journal of Health-System Pharmacy, 68(2), 143-150. https://doi.org/10.2146/ajhp100113.Development 
Deguchi, S., Yamashita, T., Igai, K., Harada, K., Toba, Y., Hirata, K., Takayama, K., \& Mizuguchi, H. (2019). Modeling of hepatic drug metabolism and responses in CYP2C19 poor metabolizer using genetically manipulated human iPS cells. Drug Metabolism and Disposition, 47(6), 632-638. https://doi.org/10.1124/dmd.119.086322

Duong, B. Q., Arwood, M. J., Hicks, J. K., Beitelshees, A. L., Franchi, F., Houder, J. T., Limdi, N. A., Cook, K. J., Owusu Obeng, A., Petry, N., Tuteja, S., Elsey, A. R., Cavallari, L. H., \& Wiisanen, K. (2020). Development of customizable implementation guides to support clinical Aaoption of pharmacogenomics: Experiences of the implementing GeNomics in pracTicE (IGNITE) Network. Pharmacogenomics and Personalized Medicine, (13), 217-226. https://doi.org/ 10.2147/PGPM.S241599

Favela-Mendoza, A. F., Martinez-Cortes, G., HernandezZaragoza, M., Salazar-Flores, J., Muñoz-Valle, J. F., Martinez-Sevilla, V. M., VelásquezSuárez, N. Y., \& Rangel-Villalobos, H. (2015). Genetic variability of CYP2C19 in a Mexican population: Contribution to the knowledge of the inheritance pattern of CYP2C19* 17 to develop the ultrarapid metabolizer phenotype. Journal of Genetics, 94(1), 3-7. https://doi.org/10.1007/ s12041-015-0477-1

Flores-Gutiérrez, S., Rodríguez-Larralde, Á., Vivenes de Lugo, M., \& Castro de Guerra, D. (2017). Distribution of polymorphisms in the CYP2C9 gene and CYP2C19/CYP2C9 haplotypes among Venezuelan populations. Annals of Human Biology, 44(2), 191-198. https://doi.org/10.1080/ 03014460.2016.1192218

Gaedigk, A., Ingelman-Sundberg, M., Miller, N. A., Leeder, J. S., Whirl-Carrillo, M., \& Klein, T. E. (2018). The pharmacogene variation (PharmVar) consortium: Incorporation of the human Cytochrome P450 (CYP) Allele Nomenclature Database. Clinical Pharmacology \& Therapeutics, 103(3), 399-401. https://doi.org/ 10.1002/cpt.910

Garrido, C., Santizo, V. G., Müllers, P., Soriano, D. R., Avila, G. B., Dean, M., \& JimenezMorales, S. (2013). Frequency of thiopurine S-methyltransferase mutant alleles in indigenous and admixed Guatemalan patients with acute lymphoblastic leukemia. Medical Oncology, 30(7), 474. https://doi.org/10.1007/s12032-013-0474-2

Gialeraki, A., Markatos, C., Grouzi, E., Merkouri, E., Travlou, A., \& Politou, M. (2010). Evaluation of a reverse-hybridization StripAssay for the detection of genetic polymorphisms leading to acenocoumarol sensitivity. Molecular Biology Reports, 37(4), 1693-1697. https://doi.org/10.1007/ s11033-009-9587-2

Gonzalez, H. M., Romero, E. M., Peregrina, A. A., Chávez, T. D. J., Escobar-Islas, E., Felipe, L. K., \& Hoyo-Vadillo, C. (2003). CYP2C19-and CYP3A4-dependent omeprazole metabolism in West Mexicans. Journal of Clinical Pharmacology, 43(11), 1211-1215. https://doi.org/ $10.1177 / 0091270003258170$

Gonzalez-Covarrubias, V., Martínez-Magaña, J. J., Coronado-Sosa, R., Villegas-Torres, B., GenisMendoza, A. D., Canales-Herrerias, P., Nicolini, H., \& Soberón, X. (2016). Exploring variation in known pharmacogenetic variants and its Association with Drug Response in Different Mexican Populations. Pharmaceutical Research, 33(11), 2644-2652. https://doi.org/10.1007/s11095016-1990-5.

Gonzalez-Covarrubias, V., Morales-Franco, M., CruzCorrea, O. F., Martínez-Hernández, A., GarcíaOrtíz, H., Barajas-Olmos, F., Genis-Mendoza, A. D., Martínez-Magaña, J. J., Nicolini, H., Orozco, L., \& Soberón, X. (2019). Variation in actionable pharmacogenetic markers in natives and mestizos from Mexico. Frontiers in Pharmacology, 10, Article 1169. https://doi.org/ 10.3389/fphar.2019.01169

Haga, S. B., \& Kantor, A. (2018). Horizon scan of clinical laboratories offering pharmacogenetic testing. Health Affairs, 37(5), 717-723. https://doi.org/ 10.1377/hlthaff.2017.1564

Hewett, M., Oliver, D. E., Rubin, D. L., Easton, K. L., Stuart, J. M., Altman, R. B., \& Klein, T. E. (2002). PharmGKB: The pharmacogenetics knowledge base. Nucleic Acids Research, 30(1), 163-165. https://doi.org/10.1093/nar/30.1.163

Ibeanu, G. C., Goldstein, J. A., Meyer, U. R. S., Benhamou, S., Bouchardy, C., Dayer, P., Blaisdell, J., \& Blaisdell, J. (1998). Identification of new 
human CYP2C19 alleles (CYP2C19* 6 and CYP2C19*2B) in a Caucasian poor metabolizer of mephenytoin. Journal of Pharmacology and Experimental Therapeutics, 286(3), 1490-1495.

Ingelman-Sundberg, M., Sim, S. C, Gómez, A., \& Rodriguez-Antona, C. (2007). Influencia de los polimorfismos del citocromo $\mathrm{P} 450$ en las terapias farmacológicas: aspectos farmacogenéticos, farmacoepigenéticos y clínicos. Farmacología y Terapéutica, 116(3), 496-526. https://doi.org/10. 1016/j.pharmthera.2007.09.004

Isaza, C., Henao, J., Isaza, J. H., Sepúlveda, J. C., \& Beltrán, L. (2006). Caracterización genotípica y fenotípica CYP2C19 de población mestiza colombiana. Revista Médica de Risaralda, 12(2), 5-13. http://dx.doi.org/10.22517/25395203.979

Kaneko, A., Lum, J. K., Yaviong, L., Takahashi, N., Ishizaki, T., Bertilsson, L., Kobayakawa, T., \& Björkman, A. (1999). High and variable frequencies of CYP2C19 mutations: Medical consequences of poor drug metabolism in Vanuatu and other Pacific islands. Pharmacogenetics, 9(5), 581-590.

Küpfer, A., \& Preisig, R. (1984). Pharmacogenetics of mephenytoin: A new drug hydroxylation polymorphism in man. European Journal of Clinical Pharmacology, 26(6), 753-759. https://doi.org/10.1007/BF00541938

Leitão, L. P., Souza, T. P., Rodrigues, J. C., Fernandes, M. R., Santos, S., \& Santos, N. P. (2020). The metabolization profile of the CYP2D6 gene in Amerindian populations: A review. Genes, 11(3), 262. https://doi.org/10.3390/genes11030262

León-Moreno, L. C., Saldaña-Cruz, A. M., SánchezCorona, J., Mendoza-Carrera, F., García-Zapién, A. G., Revilla-Monsalve, C., Islas-Andrade, S., Brito-Zurita, O., Pérez-Vargas, A., \& FloresMartínez, S. E. (2019). Distribution of potential risk alleles and haplotypes of the $C Y P 2 C 9$ and CYP2C19 genes in Mexican native populations: A comparative study among Amerindian populations. Meta Gene, 20, Article 100565. https://doi.org/10.1016/j.mgene.2019.100565

Levano, S., Ginz, H., Siegemund, M., Filipovic, M., Voronkov, E., Urwyler, A., \& Girard, T. (2005). Genotyping the butyrylcholinesterase in patients with prolonged neuromuscular block after succinylcholine. Anesthesiology: The Journal of the American Society of Anesthesiologists, 102(3), 531-535. https://doi.org/10.1097/00000542200503000-00009

López-Cortés, A., Paz-y-Miño, C., Guerrero, S., Jaramillo-Koupermann, G., Cáceres, Á. L., Intriago-Baldeón, D. P., García-Cárdenas, J. M., Guevara-Ramírez, P., Armendáriz-Castillo, I., Leone, P. E., Quiñones, L. A., Cayún, J. P., \& Soria, N. W. (2019). Pharmacogenomics, biomarker network, and allele frequencies in colorectal cancer. The Pharmacogenomics Journal, 20(4). 1-23. https://doi.org/10.1038/s41397-019-0102-4

Manikandan, P., \& Nagini, S. (2018). Cytochrome p450 structure, function and clinical significance. Current Drug Targets, 19(1). 38-54. https://doi.org/ 10.2174/1389450118666170125144557

Martinez-Espin, E., Martínez-Gonzalez, L. J., Fernandez-Rosado, F., Entrala, C., Alvarez, J. C., Lorente, J. A., Budowle, B., \& De Monroy, M. O. (2006). Guatemala mestizo population data on 15 STR Loci (Identifiler ${ }^{\circledR}$ Kit). Journal of Forensic Sciences, 51(5), 1216-1218. https://doi.org/ 10.1111/j.1556-4029.2006.00249.x

Martínez-Magaña, J. J., Genis-Mendoza, A. D., Villatoro Velázquez, J. A., Camarena, B., Martín Del Campo Sanchez, R., Fleiz Bautista, C., Bustos Gamiño, M., Reséndiz, E., Aguilar, A., Medina-Mora, M. E., \& Nicolini, H. (2020). The Identification of Admixture Patterns Could Refine Pharmacogenetic Counseling: Analysis of a Population-Based Sample in Mexico. Frontiers in Pharmacology, 11, 324. https://doi.org/10.3389/ fphar.2020.00324

Martínez-Quintana, E., Rodríguez-González, F., Medina-Gil, J. M., Garay-Sánchez, P., \& Tugores, A. (2017). Actividad de CYP2C19 y factores de riesgo cardiovascular en pacientes con síndrome coronario agudo. Medicina Clínica, 149(6), 235-239.

Mirzaev, K. B., Zelenskaya, E. M., Barbarash, O. L., Ganyukov, V. I., Apartsin, K. A., Saraeva, N. O., Nikolaev, K. Y., Ryzhikova, K. A., Lifshits, G. I., \& Sychev, D. A. (2017). CYP2C19 polymorphism frequency in Russian patients in Central Russia and Siberia with acute coronary syndrome. Pharmacogenomics and Personalized 
Medicine, 10, 107. https://doi.org/10.2147/PGPM. S126305

Naranjo, M. E. G., Rodrigues-Soares, F., Penas-Lledo, E. M., Tarazona-Santos, E., Farinas, H., Rodeiro, I., Terán, E., Grazina, M., Moya, G. E., LópezLópez, M., Sarmiento, A. P., Calzadilla, L. R., Ramírez-Roa, R., Ortíz-López, R., EstévezCarrizo, F. E., Sosa-Macías, M., Barrantes, R., Llerena, A. (2018). Interethnic variability in CYP2D6, CYP2C9, and CYP2C19 genes and predicted drug metabolism phenotypes among 6060 Ibero-and native Americans: RIBEFCEIBA consortium report on population pharmacogenomics. Omics, 22(9), 575-588. https://doi.org/10.1089/omi.2018.0114

Relling, M. V., Schwab, M., Whirl-Carrillo, M., Suarez-Kurtz, G., Pui, C. H., Stein, C. M., Moyer, A. M., Evans, W. E., Klein, T. E., AntillonKlussman, F. G., Caudle, K. E., Kato, M., Yeoh, A. E., Schmiegelow, K, Yang, J. J. (2019). Clinical pharmacogenetics implementation consortium guideline for thiopurine dosing based on TPMT and NUDT 15 genotypes: 2018 update. Clinical Pharmacology \& Therapeutics, 105(5), 1095-1105. https://doi.org/10.1002/cpt.1304

Salazar-Flores, J., Torres-Reyes, L. A., MartínezCortés, G., Rubi-Castellanos, R., Sosa-Macías, M., Muñoz-Valle, J. F., González-González, C., Ramírez, A., Román, R., Méndez, J.L., Barrera, A., Torres, A., Medina, R., \& Rangel-Villalobos, H. (2012). Distribution of CYP2D6 and CYP2C19 polymorphisms associated with poor metabolizer phenotype in five Amerindian groups and western Mestizos from Mexico. Genetic Testing and Molecular Biomarkers, 16(9), 1098-1104. https://doi.org/10.1089/gtmb.2012.0055

Schaeffeler, E., Zanger, U. M., Eichelbaum, M., AsantePoku, S., Shin, J.-G., \& Schwab, M. (2008). Highly Multiplexed Genotyping of Thiopurine

S-Methyltransferase Variants Using MALDI-TOF Mass Spectrometry: Reliable Genotyping in Different Ethnic Groups. Clinical Chemistry, 54(10), 1637-1647. https://doi.org/10.1373/ clinchem.2008.103457
Shah, R. R., Gaedigk, A., LLerena, A., Eichelbaum, M., Stingl, J., \& Smith, R. L. (2016). CYP450 genotype and pharmacogenetic association studies: A critical appraisal. Pharmacogenomics, 17(3), 259-275. https://doi.org/10.2217/pgs.15.172

Silva-Zolezzi, I., Hidalgo-Miranda, A., Estrada-Gil, J., Fernandez-Lopez, J. C., Uribe-Figueroa, L., Contreras, A., Balam-Ortiz, E., Bosque-Plata, L. del, Velazquez-Fernandez, D., Lara, C., Goya, R., Hernandez-Lemus, E., Davila, C., Barrientos, E., March, S., \& Jimenez-Sanchez, G. (2009). Analysis of genomic diversity in Mexican Mestizo populations to develop genomic medicine in Mexico. Proceedings of the National Academy of Sciences, 106(21), 8611-8616. https://doi.org/10.1073/pnas.0903045106

Sosa-Macías, M., Teran, E., Waters, W., Fors, M. M., Altamirano, C., Jung-Cook, H., Galaviz-Hernández, C., López-López, M., Ramírez, D., Moya, G., Hernández, F., Fariñas, H., Ramírez, R., Céspedes-Garro, C., Tarazona-Santos, E., \& Llerena, A. (2016). Pharmacogenetics and ethnicity: Relevance for clinical implementation, clinical trials, pharmacovigilance and drug regulation in Latin America. Pharmacogenomics, 17(16), 1741-1747. https://doi.org/10.2217/pgs-2016-0153

Spierings, G., \& Dunbar, S. A. (2013). Pharmacogenetics Using Luminex ${ }^{\circledR}$ xMAP ${ }^{\circledR}$ Technology: A Method for Developing a Custom Multiplex Single Nucleotide Polymorphism Mutation Assay. En F. Innocenti, \& R. H. N. van Schaik (Eds.), Pharmacogenomics: Methods and Protocols (pp. 115-126). Humana Press. https://doi.org/10.1007/978-1-62703-435-7_7

Wedlund, P. J. (2000). The CYP2C19 enzyme polymorphism. Pharmacology, 61(3), 174-183. https://doi.org/10.1159/000028398

Whirl-Carrillo, M., McDonagh, E. M., Hebert, J. M., Gong, L., Sangkuhl, K., Thorn, C. F., Altman, R. B., \& Klein, T. E. (2012). Pharmacogenomics knowledge for personalized medicine. Clinical Pharmacology and Therapeutics, 92(4), 414-417. https://doi.org/10.1038/clpt.2012.96 
Xajil Ramos, L. Y., Gaitán Izaguirre, G. M. E., Luna Aguilera, M. F., Vargas Rosales, R. J., Higueros Villagrán, R. A., \& Saldaña Santiago, D. G. (2020). Respuesta farmacogenética a escitalopram en pacientes geriátricos en Guatemala. Revista de la OFIL, 30(2), 121-125. https://doi.org/10.4321/ s1699-714x2020000200011

Xie, H. G., Prasad, H. C., Kim, R. B., \& Stein, C. M. (2002). CYP2C9 allelic variants: Ethnic distribution and functional significance. Advanced Drug Delivery Reviews, 54(10), 1257-1270. https://doi.org/10.1016/s0169-409x(02)00076-5
Zanger, U. M., \& Schwab, M. (2013). Cytochrome P450 enzymes in drug metabolism: Regulation of gene expression, enzyme activities, and impact of genetic variation. Pharmacology \& Therapeutics, 138(1), 103-141. https://doi.org/ 10.1016/j.pharmthera.2012.12.007

Zhang, L., Sarangi, V., Moon, I., Yu, J., Liu, D., Devarajan, S., Reid, J., Kalari, K., Wang, L., \& Weinshilboum, R. (2020). CYP2C9 and CYP2C19: Deep mutational scanning and functional characterization of genomic missense variants. Clinical and Translational Science, 13(3), 1-16. https://doi.org/10.1111/cts.12758 